\title{
Ovarian cancer: early detection, angiogenesis, lymphangiogenesis and current prospects for therapy
}

\begin{abstract}
I Chair and Department of Gynecological Oncology and Gynecology is a specialist research center providing help in diagnostics and treatment of gynecological malignancies. The research work is focused on the processes of angiogenesis and lymphangiogenesis. Development of blood and lymphatic vessels is subject to research in a wide range of malignancies, including ovarian cancer, endometrial cancer and uterine sarcomas. Angiogenesis in malignancies of the female genital tract is investigated by using some modern 3D sonography that uses high-definition blood flow imaging. Ovarian Tumors and Early Ovarian Cancer Detection unit was established in 2002 and since that time more than 3500 patients with difficult to diagnose tumors have been consulted and treated in the Department. Ovarian cancer immunology studies are the second leading research fiekld in the 1st Chair Department of Gynecological Oncology and Gynecology. The Department is well equipped with diagnostic devices as well as a scientific laboratory. This allows for studies in the fields of imaging of masses, their immunology, biochemistry and molecular biology. Understanding immunological response in patients with ovarian cancer is the key to develop new, effective therapies, including immunological vaccines. In this area we are cooperating with prominent international research centers: Department of Surgery, University of Michigan and Department of Microbiology and Immunology, University of Arkansas. Results of our research are published in both Polish and international journals specializing in fields of gynecology, oncology, immunology and basic science.
\end{abstract}

Keywords: ovarian cancer, early detection, angiogenesis, immunotherapy.

DOI: $10.1515 /$ pjph-2015-0017

\section{INTRODUCTION}

\section{Ovarian Cancer - Early Detection Program 2001-2015}

In 2001 the Department received Voluson 730 3D manufactured by the Austrian company, Kretz. It was the newest system at that time. In 2002 the ultrasound diagnostics division of the Department was subdivided into General Gynecological Ultrasound unit and specialized Ovarian Tumors and Early Ovarian Cancer Detection unit which, from that time on, has been supervised by Professor Artur Czekierdowski, an expert in ovarian pathology and a member of the International Ovarian Tumor Analysis Group (IOTA). According to the Polish National Statistical Annual Survey, during the past 15 years, there has been a significant increase in the number of cases of early ovarian cancer detection in the Lublin region. As of now, it still remains one of the highest in Poland. Our achievements in this field have been documented in several national and international publications [1-3].

Epithelial ovarian cancer (EOC) is one of the most dangerous gynecological pathologies. It happens in the majority of industrialized countries and Poland is no exception. In spite of the fact that it is much less frequent than breast cancer (with annual prevalence of approximately 16.000 new cases,) EOC with more than 3000 new cases detected each year is the most lethal genital tract malignancy taking the lives of $60-70 \%$ of the women affected by it. At the moment, detecting the earliest clinical stages of the disease seems the best way to drive the high mortality rates down [4]. Usually, these cases are unlikely to produce significant symptoms which is the main reason why the diagnosis happens too late and the complex treatment fails.

Ovarian Tumors and Early Ovarian Cancer Detection unit has undergone modernization in 2007 when we received the most sophisticated GE Voluson E8 EXPERT ultrasound system equipped with $3 \mathrm{D}$ probes and modern software. The quality of images has been improved substantially and new capabilities including "High Definition Flow" detection has proven very helpful in distinguishing vascularity of examined masses. Complementary methods that we implemented included correlation of ultrasound images with the postoperative immunohistochemistry such as CD34 and CD105 assessment of adnexal masses for new blood vessel formation as well as selected tumor marker assessment. In selected cases together with the imaging techniques such like ultrasound and CAT pelvic and abdominal scans we have used serum VEGF, CD105, PDGF and, since 2011 serum HE4 levels to better assess tumors preoperatively [3,5].

I Chair and Department of Gynecological Oncology and Gynecology, Medical University, Lublin, Poland 
In the last two years we have also included cooperation with the Clinical Genetics Unit of our Medical University for possible BRCA-1 and BRCA-2 genetic mutations detection.

For the initial EOC and other ovarian malignant lesions detection and preoperative differentiation we have been using primarily a method of subjective tumor assessment called "pattern recognition". The less experienced members of our team continue to use primarily other popular classification systems like: Risk of Malignancy Index (RMI), ROMA algorithm based on serum CA125 and HE4 assessment and IOTA ,simple rules"[6]. In difficult cases we also use more advanced classification tools like IOTA group logistic regression models 1 and 2 (LR1 and LR2) or computer generated artificial neural networks [7-9] and, since 2014 also the newest IOTA group "ADNEX" model published in BMJ in October 2014 [10]. These methods allow for correct assessment of the risk for every individual patient and a follow-up including either observation or surgery indications.

Ovarian Tumors and Early Ovarian Cancer Detection unit has not only been serving Lublin Voivodeship, with a population of approximately 2 million people, but also admitted patients from other parts of Poland, mainly south eastern part of Poland. To put it another way, the Unit provided care to each and every patient with adnexal masses looking for help. The Unit performs about 250-300 expert examinations every year. The youngest woman with malignant ovarian tumor was 17 years old, the oldest was 94 . Both of them received correct diagnoses and were successfully treated in the Department of Gynecological Oncology and Gynecology of the Medical University of Lublin. As of now, the number of women suffering from hard to diagnose adnexal masses has exceeded 3500 cases. The leading position in early EOC cases and other malignant adnexal masses detection rate provided by our Ovarian Tumors and Early Ovarian Cancer Detection unit would not have been possible, were it not for the help of many "primary care" gynecologists and other specialists based in our region and neighboring cities. They still remain the first to diagnose ovarian cancer which means they have been sending us these patients for receiving clinical expert consultation.

Ovarian cancer: angiogenesis, lymphangiogenesis and current prospects for therapy

\section{Angiogenesis}

Angiogenesis is one of the crucial processes in the development of various tumor types. The correlation between this process and patients' outcome has been investigated in many gynecological and non-gynecological malignancies. Recently, a number of studies looking at the potential role angiogenesis can play in tumor diagnostics, prognostication and therapy have been released. During the last few years, many anti-angiogenic agents were developed, therefore better understanding of angiogenesis and its role in tumors' biology seems to be essential for introduction of new therapeutic strategies.

It is crucial to understand the role played by angiogenesis in growth of malignancies and how to link this process to the clinical outcome of the disease, which, in turn, calls for the employment of qualitatively and quantitatively reliable exponents - angiogenesis markers. These tumor phenotypic qualities can be assessed with the use of immunohistochemistry what reflects angiogenesis intensity, as well as its specific neoplastic properties. Among the angiogenesis markers most frequently examined is microvessels' density. A close correlation between tumour vascularisation and its growth dynamics suggests that markers and modu-lator's of angiogenesis obtainable for research may be of significant prognostic value in predicting the clinical outcome and development of many gynecological malignancies.

During the research, we examined microvessel density using anti-CD34 and anti-CD105 antibodies. These methods are widely used to assess vascularisation of the tumor, its advantage is that results are obtained as absolute numbers (amount of vessels in square $\mathrm{mm}$ ) [11].

The objective of the research in field of angiogenesis is to seek an answer to the question of a possible practical use of the markers and modulators of angiogenesis as prognostic factors, useful in predicting the results of planned treatment and as targets for future therapeutic strategies.

Among substances playing role in angiogenesis wide range was examined in our research, i.e.:

- VEGF-A (Vascular Endothelial Growth Factor-A): one of the most important molecules in the process of angiogenesis. Its main role is to induce proliferation in endothelial cells and to inhibit its apoptosis. The mechanism of its activity is based on ligation with VEGFR receptors $[12,13]$.

- VEGF R-1, VEGF R-2 (Vascular Endothelial Growth Factor Receptor-1 and -2): transmembrane receptors, that link selectively with VEGF family. This intracellular part presents activity of tyrosine kinase and regulates metabolism of endothelial cells $[14,15]$.

- Neuropiline family: Consist of two proteins: Neuropilin-1, Neuropilin-2. These substances play role as coreceptors for VEGFRs. Furthermore neuropilines are receptors for class 3 semaphorins. Interestingly enough, neuropilins possess the ability to activate various metabolic pathways, depending of their ligands $[16,17]$.

- EGF (Epidermal Growth Factor): This factor stimulates proliferation and migration of cells by linking with its receptor EGFR that is tyrosine kinase. Cells metabolic changes induced by EGF are i.e. expression of transcription factors [18].

\section{Lymphangiogenesis}

Lymphangiogenesis is a potent process occurring during physiological condition such as embriogenesis and it ceases postpartum. In mature organisms, physiologically lymphangiogenesis is activated in very strict conditions e.g. tissue repair, inflammation. In pathological circumstances such as tumor growth (oncogenesis), excessive proliferation and an occurrence of new vessel sprouting. Cancer-induced lymphangiogenesis is triggered, promoted and executed by many different growth factors [19]. They are produced and released not only by the tumors themselves, but also by microenvironment that includes stromal and tumorinfiltrating cell [20]. The most important pathological role of lymphangiogenesis is promotion of tumor growth and induction of cancer metastasis. The invasion of tumor cells into lymphatic vessels is an important step during carcinogenesis and, 
consequently, leads to lymph nodes and distant metastases. It favors disease progression and affects the prognosis. For many years, tumor-induced lymphangiogenesis has been in the shadow of angiogenesis largely due to the lack of any suitable markers distinguishing vascular epithelium from lymph vessel.

The regulation of the lymphangiogenesis is very complex and still has yet to be fully understood. Similarly to angiogenesis, the family of VEGF proteins plays an important role in lymphangiogenesis regulation. Process of linking VEGF$\mathrm{C}$ and VEGF-D to their receptor VEGFR-3 seems to be crucial for appropriate lymphangiogenesis. Nowadays a lot of factors that regulates lymphangiogenesis were recognized. The most important among these factors are: IGF (insulin-like growth factor), HGF (hepatocyte growth factor), angiopoetin, endotelin-1, FGF-2 (fibrobast growth factor -2), PDGF (platelet derived growth factor). PROX-1 (prosperohomeobox protein 1) [21].

In our center, Lymphangiogenesis is the subject of research carried out with the use of ovarian cancer uterine sarcoma and endometriosis.

Research mentioned above is based on immunochemistry with antibodies against the following substances:

- PROX-1 (Prospero homeobox protein 1): Transcription factor considered to be one of the earliest lymphatic cells markers. Its role is to catalyze expression of lymphatic cell phenotype [22,23].

- VEGFR-3 (Vascular Endothelial Growth Factor Receptor-3): Another member of VEGF receptors family. Unlikely to VEGFR-1 and 2 expressed mostly in lymphatic endothelial cells. Its activity is related to endothelial cells migration [24].

- LYVE-1 (Lymphatic Vessel Endothelial Hyaluronian Receptor-1): In lymphatic cell metabolism it plays a role in hyaluronian acid binding. LYVE-1 is one of the most specific markers of lymphatic vessels. Similarly to CD34, CD105 in blood vessels and PROX-1 in lymphatic vessels it in widely used to asses lymphatic vessels density [25].

- Podoplanin: Another marker of lymphatic endothelial cells. The exact role of podoplanin in cell metabolism was not determined so far. This marker is suspected to play role in formation of both lymphatic and blood vessels [26].

Markers of lymphangiogenesis, like those involved in the process of angiogenesis, might be useful in both prognostication and for targeted treatment on neoplasms. The aim of our studies in the field of angiogenesis is to confirm, whether investigated markers are suitable to play such roles in the clinical approach to gynecological cancer.

\section{Immunotherapy}

Classical schemes of ovarian cancer treatment rely on the surgical removal of the tumor and subsequent chemotherapy [27]. The most frequently applied schemes of chemotherapy are taxanes in connection with cisplatin or carboplatin, repeated every 21 days [28]. However, chemotherapy applied in advanced ovarian cancer does not guarantee recovery, and only slightly improves the patients' lifespan. The percentage of survivals of five-year-olds amounts only to about $20-25 \%$ tumors in the III level of clinical advancement according to FIGO and only 5\% in the IV level of the disease [29]. An alternative to the treatment of patients with ovarian cancer seems to be anti-cancerous immunotherapy, whose main aim is to activate the patient's defense mechanisms that destroys cancerous cells. In the so-far applied attempts of ovarian cancer therapy, monoclonal antibodies were applied, directed at antigens related with the neoplasm: anty-CA125; Oregovomab-MabB43.13; anty-HER-2;Trastuzumab, antyMUC-1;HMFG1, anti-idiotypic antibodies (anty-idiotypic Abagovomab; ACA-125), cytokines (IL-2, IFN- $\alpha$, IFN- $\gamma$ ) and vaccines [30-35]. Anti-neoplastic vaccines may rely on the use of peptides, whole cancerous cells, shock proteins and dendritic cells (DCs) [36]. The use of DCs in the ovarian cancer therapy brings new hopes.

Anti-cancerous vaccines on the base of DCs constitute a form of adoptive immunotherapy, in which the cells of the immunological system activated beyond the patient's body with cancerous antigens are used for fighting the neoplasm. The following neoplastic antigens trigger an immunological reaction in ovarian cancer patients: HER2/neu, MUC1, OA3, CA-125, receptor $\alpha$ of folic acid, TAG-72,mesothelin, NY-ESO-1, sialyl-Tn [37-45].

The in vitro generation process of DCs in vitro consists of several stages: the isolation of progenitor cells, the stimulation of their differentiation into immature DCs, stimulation with neoplastic antigens, and then induction of dendritic cells maturation. The DCs generated in this way are then administered to patients as a vaccine [46]. The main aim of immunotherapy with the use of DCs is the induction of specific and effective immunological response against neoplastic antigens. The source of dendritic cells may be stem cells $\mathrm{CD}_{3} 4^{+}$isolated from umbilical cord blood, blood or bone marrow, as well as monocytes of the blood [47]. The $\mathrm{CD} 34^{+}$differentiate into DCs under the influence of cytokines that is GM-CSF and TNF $\alpha$ [48]. The enrichment of culture medium with other factors that is SCF (stem cell factor) and Flt3 ligand (Flt3L) increases the percentage of circulating DCs and intensifies the immunological response.

The most frequently used source of DCs are blood monocytes $\left(\mathrm{CD} 14^{+}\right)$. It was shown in vitro that monocytes stimulated with recombined human (rh) cytokines, that is rhGMCSF and rhIL-4/IL-13, may be transformed into immature dendritic cells capable of detecting and processing the antigen [47]. The immature DCs obtained in this way, might be stimulated with neoplastic antigens that allows for obtaining specialized cells presenting the antigen. Under the influence of rhTNF $\alpha$ and/or CD40L they are transformed into mature cells, capable of presenting the antigen and stimulating naive lymphocytes $\mathrm{T}$ [49].

To stimulate DCs, lysates of neoplastic cells, mRNA, cDNA or apoptotic bodies obtained out of them are used [46]. Nevertheless, antigens like EGF receptors: HER2 (cerbB2,HER2/neu), neoplastic and testicular antigens: NY-ESO-1, serine proteases that is the stratum corneumchymotryptic enzyme (SCCE), hepsin, matriptase, epithelial mucins: MUC1 (peptides M1.1 and M1.2), MUC2, MUC4, MUC5AC or other antigens: antigen Ca125, protein 
p53, mesothelin, HE4 [50] appear to be an interesting field to research into for ovarian cancer immunotherapy [50].

The development of antigen-specific response with the participation of DCs was observed even in patients with advanced ovarian cancer. It was proven that DCs stimulated with tumor lysates, eluated peptides or DCs hybrids with tumor cells were inducing HLA (human leukocyte antigen) of I class-dependent cytotoxic response against autologictumor cells [51].

There are not too many clinical reports concerning the immunotherapy of ovarian cancer with the use of DCs. In case of most patients previously treated with cellular immunotherapy DCs, we proved an induction of immune response, yet, this led to a clinical response only in some patient. The lack of effectiveness in anti-neoplastic immunotherapy, despite arousing immunological response, may result from the regulatory cell populations: regulatory $\mathrm{T}$ lymphocytes, suppressor cells of myeloid origin, DCs subpopulations of tolerogenic properties, or tumor associated macrophages (TAMS) [50]. Hence, the use of DCs in the ovarian cancer immunotherapy requires modification. The search for antigens specific for a given malignant tumor (TSA) is of significant importance for the improvement of immunotherapy results.

Furthermore, it is considered that vaccines with DCs should be directed against many antigens related with neoplastic tissue. We also recommend the optimization of the conditions of DCs culture. Moreover, the vaccines should be preceded with the administration of preparations eliminating regulatory $\mathrm{T}$ lymphocytes that is monoclonal antibody anti-CD25, ONTAK- hybrid protein composed of fiber toxin and IL-2and inhibiting their activity (antibody anti-CTLA-4, inhibitors of dioxygenase 2,3-indoleamin (IDO) [52]. According to the latest reports, the effectiveness of the immunotherapy may be increased through the connection of immunotherapy with conventional chemotherapy or targeted therapy. The goal of numerous researches is to draw up an effective and safe method of elimination of regulatory cell population and overcome the immunosuppressive influence of the neoplastic disease. Hopefully, the results obtained would lead to the point where immunotherapy would no longer be an experimental treatment but find its way into the routine clinical practice.

\section{REFERENCES}

1. Czekierdowski A. Studies on angiogenesis in the benign and malignant ovarian neoplasms with the use of color and pulsed Doppler sonography and serum CA-125, CA-19.9, CA-72.4 and vascular endothelial growth factor measurements. Ann Univ Mariae Curie Sklodowska Med. 2002;57:113-31.

2. Czekierdowski A, Smoleń A, Bednarek W, Kotarski J. Three dimensional sonography and 3D power angiography in differentiation of adnexal tumors. Ginekol Pol. 2002;73:1061-70.

3. Czekierdowski A, Szymański M, Szumiło J, Kotarski J. Color Doppler blood flow measurements and microvessel density assessment in ovarian tumors. Ginekol Pol. 2003;74:695-700.

4. Timmerman D, Testa AC, Bourne T, et al. International Ovarian Tumor Analysis Group. Logistic regression model to distinguish between the benign and malignant adnexal mass before surgery: a multicenter study by the International Ovarian Tumor Analysis Group. J Clin Oncol. 2005;23:8794-801.
5. Czekierdowski A, Czekierdowska S, Danilos J, et al. Microvessel density and $\mathrm{CpG}$ island methylation of the THBS2 gene in malignant ovarian tumors. J Physiol Pharmacol. 2008;59 (Suppl 4):53-65.

6. Valentin L, Ameye L, Savelli L, et al. Adnexal masses difficult to classify as benign or malignant using subjective assessment of gray-scale and Doppler ultrasound findings: logistic regression models do not help. Ultrasound Obstet Gynecol. 2011;38:456-65.

7. Daemen A, Valentin L, Fruscio R, et al. Improving the preoperative classification of adnexal masses as benign or malignant by second-stage tests. Ultrasound Obstet Gynecol. 2011;37:100-6.

8. Timmerman D, Van Calster B, Testa AC, et al. Ovarian cancer prediction in adnexal masses using ultrasound-based logistic regression models: a temporal and external validation study by the IOTA group. Ultrasound Obstet Gynecol. 2010;36:226-34

9. Testa A, Kaijser J, Wynants L, Fischerova, et al. Strategies to diagnose ovarian cancer: new evidence from phase 3 of the multicentre international IOTA study. Br J Cancer. 2014;111:680-8.

10. Van Calster B, Van Hoorde K, Valentin L, et al. Diagnosing ovarian cancer using the ADNEX risk model: A diagnostic study to differentiate between benign, borderline, stage I invasive, advanced stage invasive, and secondary metastatic tumours. Br Med J. 2014;349:g5920.

11. Dharmalingam P, Roopesh Kumar VR, Verma SK. Vascular endothelial growth factor expression and angiogenesis in various grades and subtypes of meningioma. Indian J Pathol Microbiol. 2013;56:34954.

12. Otrock ZA, Makarem JA, Shamseddine AI. Vascular endothelial growth factor family of ligands and receptors: Review. Blood Cells Mol Dis. 2007;38:258-268.

13. Wang Y, Yao X, Ge J, et al. Can vascular endothelial growth factor and microvessels density be used as prognostic biomarkers for colorectal cancer? A systematic review and meta-analysis. Scientific World J. 2014;2014:102736.

14. Matsui Y, Amano H, Ito Y, et al The role of vascular endothelial growth factor receptor-1 signaling in compensatory contralateral lung growth following unilateral pneumonectomy. Lab Invest; 2015.

15. Lu W, Li P, Shan Y, et al. Discovery of biphenyl-based VEGFR-2 inhibitors. Part 3: Design, synthesis and 3D-QSAR studies.Bioorg Med Chem. 2015;23(5):1044-54.

16. Wild J, Staton C, Chapple K, Corfe B. Neuropilins: expression and roles in the epithelium. Int J Exp Pathol.2012;93(2):81-103.

17. Kärpänen T, Heckman CA, Kesktitalo S, et. al. Functional interaction of VEGF-C and VEGF-D with neuropilin receptors. FASEB J. 2006;20:1462-72.

18. Harris RC, Chung E, Coffey RJ. EGF receptor ligands. Exp Cell Res. 2003;284(1):2-13.

19. Li S, Li Q. Cancer stem cells, lymphangiogenesis, and lymphatic metastasis. Cancer Letters. 2015;357:438-47.

20. Ji RC. Lymphatic endothelial cells, tumor lymphangiogenesis and metastasis: New insights into intratumoral and peritumoral lymphatics. Cancer Metastasis Rev. 2006;25(4):677-94.

21. He Y, Rajantie I, Pajusola K, et al. Vascular endothelial cell growth factor receptor 3-mediated activation of lymphatic endothelium is crucial for tumor cell entry and spread via lymphatic vessels. Cancer Res. 2005;65(11):4739-46.

22. Wigle JT, Oliver G. Prox1 function is required for the development of the murine lymphatic system. Cell. 1999;98(6):769-78.

23. Van der Auwera I, Cao Y, Tille JC. First international consensus on the methodology of lymphangiogenesis quantification in solid human tumours. BJC. 2006;95:1611-25.

24. Potente M, Gerhardt H, Carmeliet P. Basic and therapeutic aspects of angiogenesis.Cell. 2011;146:873-87.

25. Gu B, Alexander JS, Gu Y, et al. Expression of lymphatic vascular endothelial hyaluronan receptor-1 (LYVE-1) in the human placenta. Lymphatic Res Biol. 2007;4(1):11-7.

26. Omachi T, Kawai Y, Mizuno R, et al. Immunohistochemical demonstration of proliferating lymphatic vessels in colorectal carcinoma and its clinicopathological significance. Cancer Lett. 2007;246(1-2):167-72.

27. Kędzia H. Pierwotne nabłonkowe nowotwort jajnika. In: $H$. Kędzia. Nowotwory narządów płciowych kobiety. Poznań: Ośrodek Wydawnictw Naukowych; 1997. p. 43-81.

28. Neijt J, Engelholm S, Tuxen M, et. al. Exploratory phase III study of paclitaxel and cisplatin versus paclitaxel and carboplatin in advanced ovarian cancer. J Clin Oncol. 2000;18:3084-92. 
29. Ozols R, Rubin S, Thomas G, et. al. Epithelial ovarian cancer. In: W. Hoskins, C. Perez, R. Young. Principles and Practice of Gynecologic Oncology. Philadelphia, USA: Lippincott, Williams and Wilkins; 2000. p. 981-1057.

30. Reinartz S, Köhler S, Schlebusch H, et. al. Vaccination of patients with advanced ovarian carcinoma with the anti-idiotype ACA125: immunological response and survival (phase Ib/II). Clin Cancer Res. 2004;10:1580-87.

31. Helen T, Hoskins P, Miller D, et. al. A pilot phase 2 study of oregovomab murine monoclonal antibody to CA125 as an immunotherapeutic agent for recurrent ovarian cancer. Int J Gynecol Cancer. 2005;15:1023-34.

32. Agus D, Gordon M, Taylor C, et. al.. Phase I clinical study of pertuzumab, a novel HER dimerization inhibitor, in patients with advanced cancer. J Clin Oncol. 2005;23:2534-43.

33. Nicholson S, Bomphray C, Thomas H, et. al. A phase I trial of idiotypic vaccination with HMFG1 in ovarian cancer. Cancer Immunol Immunother. 2004;53:809-16.

34. Berek J, Hacker N, Lichtenstein A, et. al. Intraperitoneal recombinant alpha 2-interferon for 'salvage' immunotherapy in persistent epithelial ovarian cancer. Cancer Treat Rev. 1985;12 (Suppl. B):23-32.

35. Odunsi K, Qian F, Matsuzaki J, et. al. Vaccination with an NY-ESO-1 peptide of HLA class I/II specificities induces integrated humoral and $\mathrm{T}$ cell responses in ovarian cancer. Proc Natl Acad Sci USA. 2007; 104:12837-42.

36. Liu B, Nash J, Runowicz C, et. al. Ovarian cancer immunotherapy: opportunities, progresses and challenges. J Hematol Oncol. 2010;3:7.

37. Disis M, Gooley T, Rinn K, et. al. Generation of T-cell immunity to the HER-2/neu protein after active immunization with HER-2/neu peptidebased vaccines. J Clin Oncol. 2002;20:2624-32.

38. Vlad A, Kettel J, Alajez N, et. al. MUC1 immunobiology: from discovery to clinical applications. Adv Immunol. 2004;82:249-93.

39. Campbell I, Freemont P, Foulkes W, et. al. An ovarian tumor marker with homology to vaccinia virus contains an IgV-like region and multiple transmembrane domains. Cancer Res. 1992;52:5416-20.

40. Kenemans P. CA 125 and OA 3 as target antigens for immunodiagnosis and immunotherapy in ovarian cancer. Eur J Obstet Gynecol Reprod Biol. 1990;36:221-8.
41. Coliva A, Zacchetti A, Luison E, et. al. 90Y Labeling of monoclonal antibody MOv18 and preclinical validation for radioimmunotherapy of human ovarian carcinomas. Cancer Immunol Immunother. 2005;54:120013.

42. Rosenblum M, Shawver L, Marks J, et. al. Recombinant immunotoxins directed against the c-erb-2/HER2/neu oncogene product: in vitro cytotoxicity, pharmacokinetics, and in vivo efficacy studies in xenograft models. Clin Cancer Res. 1999;5:865-74.

43. Chang K, Pastan I. Molecular cloning of mesothelin, a differentiation antigen present on mesothelium, mesotheliomas, and ovarian cancers. Proc Natl Acad Sci USA. 1996;93:136-40.

44. Odunsi K, Jungbluth A, Stockert E, et. al. NY-ESO-1 and LAGE-1 cancer-testis antigens are potential targets for immunotherapy in epithelial ovarian cancer. Cancer Res. 2003;63:6076-83.

45. Sandmaier B, Oparin D, Holmberg L, et. al. Evidence of a cellular immune response against sialyl-Tn in breast and ovarian cancer patients after high-dose chemotherapy, stem cell rescue, and immunization with TheratopeSTn-KLH cancer vaccine. J Immunother. 1999;22:54-66.

46. Dallal R, Lotze M. The dendritic cell and human cancer vaccines. Curr Opin Immunol. 2000;12:583-8.

47. Fong L, Engleman E. Dendritic cells in cancer immunotherapy. Annu Rev Immunol. 2000;18:245-73.

48. Avigan D. Dendritic cells: development, function and potential use for cancer immunotherapy. Blood Rev. 1999;13:51-64.

49. Tarte K, Klein B. Dendritic cell-based vaccine: a promising approach for cancer immunotherapy. Leukemia. 1999;13:653-63.

50. Chiriva-Internati M, Mirandola L, Kast W, et. al. Understanding the cross-talk between ovarian tumors and immune cells: mechanisms for effective immunotherapies. Int Rev Immunol. 2011;30:71-86.

51. Gong J, Nikrui N, Chen D, et. al. Fusions of human ovarian carcinoma cells with autologus or allogeneic dendritic cells induce antitumor immunity. J Immunol. 2000;165:1705-11.

52. Kandalaft L, Singh N, Liao J, et. al. The emergence of immunomodulation: Combinatorial immunochemotherapy opportunities for the next decade. Gynecol Oncol. 2010;116:222-33.

\section{Corresponding author}

Jan Kotarski

16 Staszica Str., 20-081 Lublin

tel. 8153278 47, fax. 815320608

E-mail:jan.kotarski@umlub.pl 